\title{
Printed Light Tags and the Magic Bookmark
}

Using light to augment paper objects

\section{Georgios Bairaktaris}

University of Surrey, Guildford, Surrey, United Kingdom, g.bairaktaris@surrey.ac.uk

David M. Frohlich

University of Surrey, Guildford, Surrey, United Kingdom, d.frohlich@surrey.ac.uk

\section{Radu A. Sporea}

University of Surrey, Guildford, Surrey, United Kingdom, r.a.sporea@surrey.ac.uk

Paper books offer a unique physical feel, which supports the reading experience through enhanced browsing, bookmarking, freeform annotations, memory and reduced eye strain. In contrast, electronic solutions, such as tablets and e-readers, offer interactive links, updatable information, easier content sharing, and efficient collaboration. To combine the best aspects of paper and digital information for reading, we demonstrate two mechanisms for augmenting paper with light sensors that trigger digital links on a nearby smartphone. Light Tags on every page of a book are used in a first demonstration to identify which pages are open. These are replaced with an electronic Magic Bookmark in a second demonstration, avoiding the need to instrument every page.

CCS CONCEPTS - Hardware $\rightarrow$ Communication hardware, interfaces and storage $\rightarrow$ Sensor devices and platforms • Human-centered computing $\rightarrow$ Human computer interaction (HCI) $\rightarrow$ Interaction devices

Additional Keywords and Phrases: Augmentation, Paper, User Interaction, Design for Manufacture

ACM Reference Format:

Georgios Bairaktaris, David M. Frohlich, Radu A. Sporea. 2021. Printed Light Tags and the Magic Bookmark: Using light to augment paper objects. in Computer Human Interaction CHI 2021 interactivity track, May 8-13, 2021, online virtual conference.

\section{INTRODUCTION}

The value of paper and printed information is challenged by the features offered by digital media. Screen-based devices, which are used to consume rich digital content, provide attractive alternatives to paper-based information [1]. These solutions (tablets, e-readers, etc.) offer internet access, simplify content sharing, and allow for effortless collaborative annotation. Additionally, electronic devices can store a large library of books and publications in a small form-factor device, which can be carried by users with ease. These features challenge the existence of printed information. However, paper-based content has unique qualities that cannot be replaced by digital media.

Paper's unique physical feel improves various aspects of reading against the equivalent digital experience. For example, reading printed content on paper offers better comprehension, improves browsing and bookmarking, 
allows for free-form annotations, and improves collaboration [2-6]. Furthermore, research suggests that the better positional indexing and improved reading experience of paper-based books improves memory and recollection of information [7]. This has also been linked to the use of physical paper for training from a young age, which is the default media in schools [7]. In addition, the visual fatigue caused by screen-based electronic devices often drives people to the use of paper for longer reading sessions [8]. Despite the unique properties of paper, however, the use of digital media is unavoidable and the coexistence of the two types of content needs to persist [9].

The seamless integration of digital and printed media, and the interaction between them, is a challenge that has not been sustainably addressed. Multiple groups have attempted to provide a solution to this problem. Some significant contributions will be discussed in the next section. Then, our Next Generation Paper ecosystem will be introduced as a generic method for authoring and playing back links between paper books and screen-based content, together with two techniques for automatically sensing page identity using light.

\section{RELATED WORK}

The first attempt at digitally augmenting paper dates back three decades, with Wellner's Digital Desk [10]. The system enables interactions with pens and fingers on physical and digital files. It is based on an overhead camera and projector, with an image processing system that tracks reading actions on a desk. The size and cost of the hardware and its limitations in portability make it unscalable for manufacturing. Since then, core technologies of augmented paper - 1. Augmented Reality (AR) systems and 2. Internet-of-Thing (IoT) devices - have evolved.

AR systems utilize the optical recognition of printed markers to trigger actions on an adjunct device, which is used to consume the augmentation [11]. These include barcodes, QR codes, or the Anoto dot pattern read by optical reading devices or pens. Systems in this category include Webstickers [12], Books with Voices [13], ButterflyNet [14], Magic Book [15], Memento [16] and PaperClip [17]. As image recognition has improved, systems recognize photographs or pages using a webcam or camera phone, such as the Audiophoto desk [18]. One limitation of AR systems is that they require scanning actions over paper, which may be awkward when reading.

IoT approaches provide more seamless interactions, utilizing sensors in paper to trigger content by interacting with it. These include RFID tags, magnets, functional inks, buttons, or capacitive sensors. Examples include the ListenReader [19], Paper++[20], the Interactive Newsprint [21], an e-Reader paper interface [22] and TorBook [23].

Many of the above systems are handmade, expensive, and difficult to manufacture at scale. On the other hand, organic photovoltaic (OPV) materials can be treated as inks, which opens up the possibility of printing them directly on paper. Furthermore, we noticed that on physical books, only the page being read is open to light. This led Frohlich, Sporea and Calic to develop the notion of Light Tags in 2014, and build an unpublished proof-of-concept demonstrator with off-the-shelf photodiodes on each page, and a Bluetooth chip in the binding of an Audiophotobook [24]. Turning the pages of the photobook triggers a soundscape for each open page. While the interaction was magical, the implementation was not. It involved complex wiring of each page to the embedded chip through the spine of the book. In the following demonstrations, we report two subsequent implementations, which begin to show the value of light sensors for augmenting paper objects in a more scalable and sustainable way.

\section{OUR APPROACH}

In this work we are describing two hardware solutions, which can seamlessly augment printed paper books. The systems are designed within the Next Generation Paper (NGP) project [25, 26], addressing the augmentation of paper with a single ecosystem (Figure 1) optimized for producing, consuming, and maintaining digital content 
associated with a printed book. The technology, developed in collaboration with book publishers, is optimized for existing publishing workflows. In fact, augmented books, or ' $a$-books' for short, are authored as interactive e-books in EPUB3 format, either in a professional book layout system (e.g Adobe InDesign) or in the NGP Authoring App. The printed part of the interactive e-book can trigger its hotlinks on a nearby smartphone using an NGP Player App.

Two techniques for triggering hotlinks are supported within the NGP Player App. These are centered around the physical book and its handling as the main interface to the digital world. In what we refer to as "second generation (2G) paper", an image recognition engine identifies page content and links to the relevant augmentation media. This is an AR approach. In the "third generation (3G) paper", which is the focus of this demonstrator, a hybrid electronic device combining printed and commercial electronics is developed. This is an IoT approach. The device automatically detects the active page and triggers digital content on a nearby display, thus maintaining a more natural interaction with the book. Based on user studies of the $2 \mathrm{G}$ technology, the a-book added further depth to the written material (through complementary multimedia), which ultimately improved the reading experience [27].
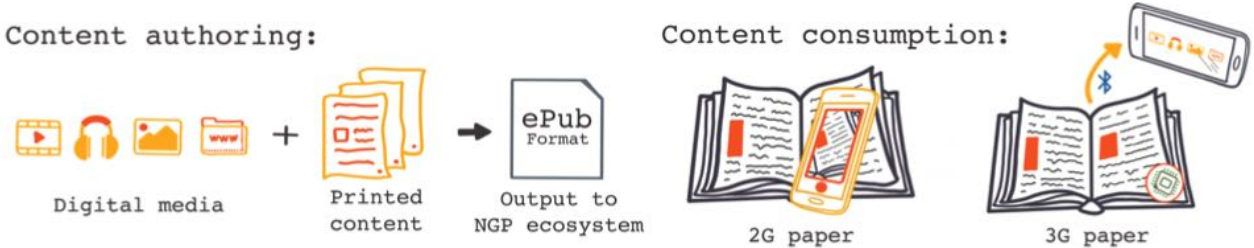

Figure 1: The Next Generation Paper (NGP) ecosystem. Digital content is associated with printed information, exported to an ePub file, and consumed by commercial e-book readers or imported to the NGP devices

\section{DEMONSTRATORS}

\subsection{Demonstrator 1: Printed light tags}

Two demonstrators are presented here. The first demonstrator is an augmented travel guide using Light Tags; a 3G technology prototyped in the NGP project [27]. This hybrid electronic device, which looks like a typical hardcover book (Figure 2), uses commercial and printed electronics embedded in the pages and cover to enable interactivity.

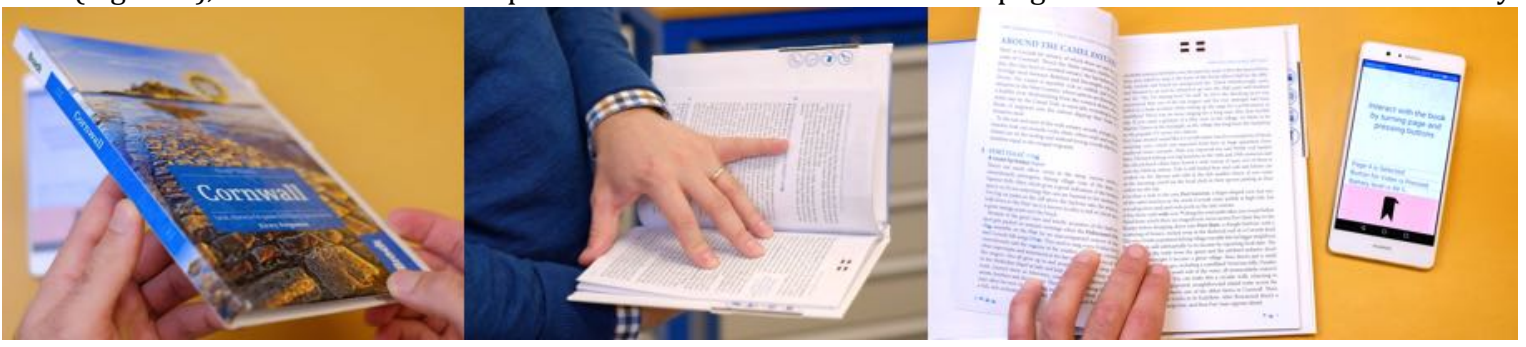

Figure 2: The first demonstrator, the Light Tags, is used in the $3 \mathrm{G}$ a-book device for open page detection. Pressing the buttons on the side of the book triggers the corresponding digital media on the phone connected to the hybrid device.

While handling the book, the reader uses touch sensitive areas to trigger available media, identified by icons on each page. The electronics automatically recognize which page is being read and transmits the page number and media type desired to a nearby smart device that runs an NGP proprietary application. The augmentation media can be consumed in parallel with the printed content, by interacting solely with the a-book. The system comprises two parts: 1 . An automatic page detection device (referred to as Light Tags), and 2. the control electronics. 
The Light Tags use printed Organic Photovoltaics (OPVs) (Figure 3), laminated between two sheets of paper to hide them from the user and to maintain the feeling of paper. They generate a unique code for each set of open pages which is relayed to the control electronics via the spine. Power consumption is negligible, as the scheme uses the photogenerated voltage for page number detection and, as the sensors are fully printed on a roll-to-roll system, the cost is low. Further advances in printed electronics will enable depositing the sensors directly on the pages.

The control electronics, including page number interpretation, user input, power management and communication, are hidden within the hard cover of the device. Data pathways between the sensors on each page and the control electronics are realized by screen-printing on a plastic substrate, attached to the inside of the cover, in a streamlined assembly process consistent with large-scale manufacturing. With the above design approach, a pure book feel is maintained, and the interaction with the book becomes seamless and familiar.

This promising approach has some inherent technical limitations. Printing OPVs on paper remains a challenging topic, as moisture sensitivity reduces active material lifetime and, currently, assembly and wiring of the sensors within the pages is a manual process which cannot be automated. From an interaction perspective, the laminated pages are thicker than one would expect from a book, which reduces usability. Finally, the hidden electronics in the cover of the device limit the application scope of this technology; only hardcover books can be augmented this way.
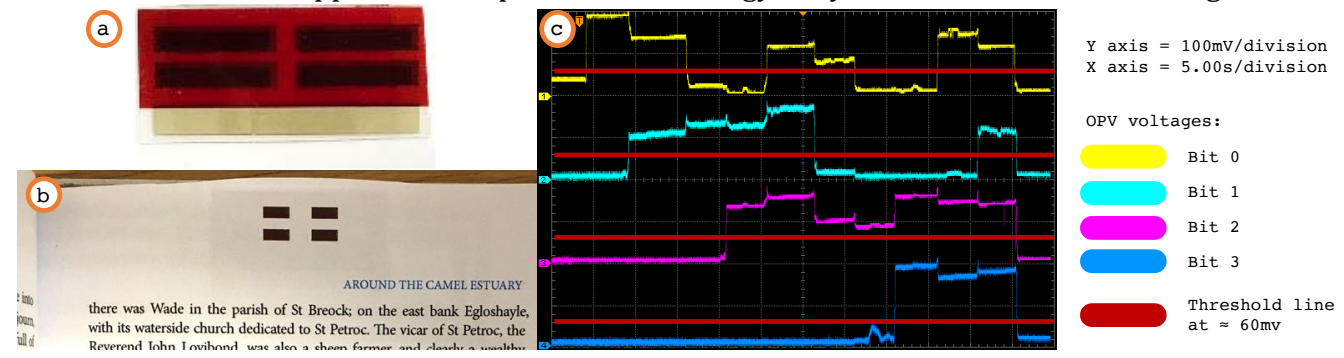

Figure 3: Light Tags details. Flexible photovoltaic modules (a) are laminated between two paper pages (b). Each module has 4 sensors that create a unique binary code per page. An oscilloscope capture of this code while flipping the pages of the book is shown in c.

\subsection{Demonstrator 2: Magic Bookmark}

The second demonstrator is an augmented travel booklet with a Magic Bookmark. This provides solutions to both technical and interaction challenges of the printed Light Tags above. An early prototype of the technology is implemented on a soft-cover chapter of the Cornwall travel guide above. The device is augmented with sensors that automatically detect the open page, but can be used as a typical bookmark. Near the book's spine, each page contains a unique binary code, discerned by the electronically enhanced bookmark and transmitted to an adjunct device that runs the NGP application (the concept is shown in Figure 4a). The control electronics are of the same complexity as with the Light Tags, and can be attached as a separate device on compatible books, or incorporated in a generic cover. Bailey et al. [28] uses a functionally similar approach for synchronizing paper- and screen-based versions of books, still requiring that every page is augmented with conductive tags which represent a unique page number.

Various methods have been proposed for realizing automatic page detection, with varying degrees of technical complexity, user convenience, and streamlined manufacturing of the book's pages. The chosen method was utilizing optical cut-outs (punch-holes) that represent a unique binary code for each page (demonstrated in Figure 4b). The augmented bookmark is populated with a series of photodiodes, and is positioned on the page following the one that is currently being read by the user. The photodiodes are used in photovoltaic mode, and the photogenerated voltage of each diode is read by the control electronics. A reference sensor at the top of the bookmark always 
remains exposed through an opening on the paper. Each voltage is compared against the reference to distinguish diodes directly exposed to light from ones covered by paper. The control electronics responsible for translating the optical binary code and transmitting it to the proprietary application can either be combined with the bookmark, as a separate device, or incorporated within the book's cover. Pictures of the prototype can be seen in Figure 5.

This technique offers robust and reliable open-page recognition and improved abstraction from the reader's view. It eliminates the need to instrument every page, greatly simplifying the manufacturing process. Compared to the Light Tags and other platforms in literature, the Magic Bookmark creates a more natural interaction. However, placing the bookmark on the next page differs slightly from a typical interaction with bookmarks and deviates from the zero-delta concept (no change in behaviour), which is a priority for our work. The interaction, in combination with the NGP's ecosystem for publishing, linking and maintaining the augmentation content, creates a robust and complete solution to the coexistence of physical books and digital media, without compromising the paper feel.

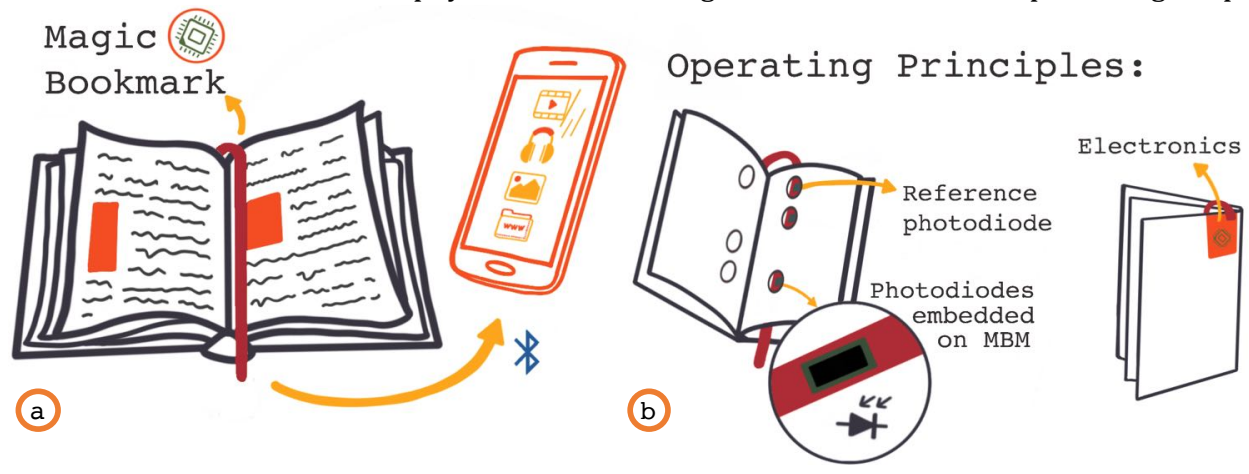

Figure 4: The Magic Bookmark concept (part a) and the main operating principles (part b).

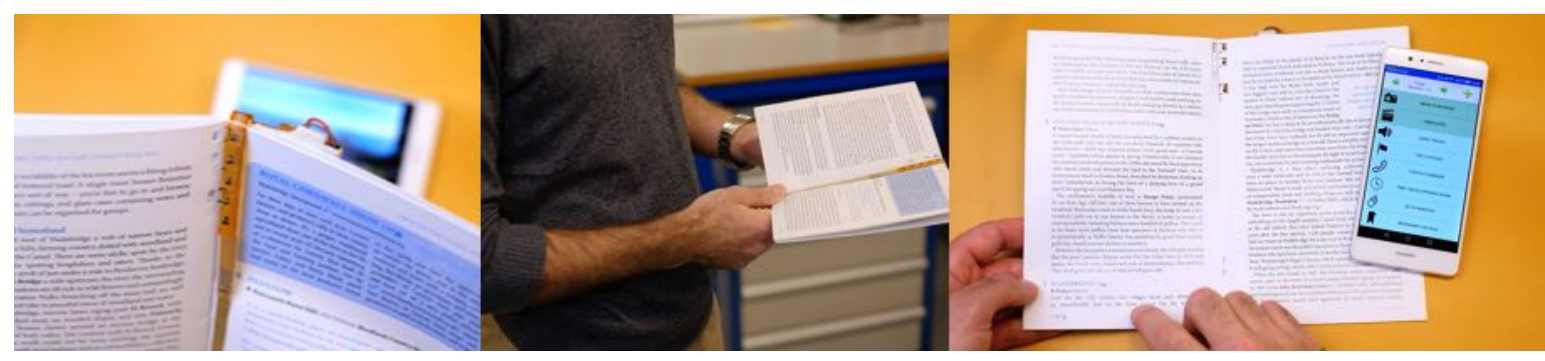

Figure 5: The second demonstrator, the Magic BookMark, in use. This prototype is utilizing the first method for open page detection. The bookmark is placed behind the active page and the content consumption is handled by the Next Generation Paper ecosystem.

\section{CONCLUSIONS}

The seamless coexistence of paper and multimedia content will change the way people consume information and interact with books. Our demonstrators introduce a sensor platform that augments physical books with digital information, using naturally incident light to detect user interest and interaction. The Next Generation Paper ecosystem supports authoring and maintaining the links between content types. This solution has been well received by users and, while the 3G technology lacks commercial maturity, the Magic Bookmark addresses manufacturability problems. Our approach provides a link to the digital world via handling solely a physical book. The interaction is natural, and the electronics are abstracted from the user, maintaining the bookness of the device. 


\section{ACKNOWLEDGMENTS}

The Next Generation Paper Project EP/P02579X/1 was funded by the Engineering and Physical Sciences Research Council. The authors thank the project team, Surrey's Undergraduate Electronics Lab staff, Dr Brice Le Brogne, Dr Lory Liza Bulay-og, VTT, and NovaCentrix for their contributions to the demonstrators.

\section{REFERENCES}

[1] Beat Signer and Moira C. Norrie. 2010. Interactive Paper: Past, Present and Future. Proceedings of PaperComp 2010, 1st International Workshop on Paper Computing: 10-13.

[2] Dennis T Clark, Susan P Goodwin, Todd Samuelson, and Catherine Coker. 2008. A qualitative assessment of the Kindle e-book reader: results from initial focus groups. Performance Measurement and Metrics 9, 2: 118-129.

[3] Abigail J Sellen and Richard H R Harper. 2003. The myth of the paperless office. MIT press.

[4] David M Levy. 2012. Scrolling forward: Making sense of documents in the digital age. Simon and Schuster.

[5] Anne Mangen, Bente R Walgermo, and Kolbjørn Brønnick. 2013. Reading linear texts on paper versus computer screen: Effects on reading comprehension. International journal of educational research 58: 61-68.

[6] Hanho Jeong. 2012. A comparison of the influence of electronic books and paper books on reading comprehension, eye fatigue, and perception. The Electronic Library.

[7] Sara J. Margolin, Casey Driscoll, Michael J. Toland, and Jennifer Little Kegler. 2013. E-readers, computer screens, or paper: Does reading comprehension change across media platforms? Applied Cognitive Psychology 27, 4: 512-519.

[8] Simone Benedetto, et al. 2013. E-readers and visual fatigue. PLoS ONE 8, 12.

[9] Yin Zhang and Sonali Kudva. 2014. E-books versus print books: Readers' choices and preferences across contexts. Journal of the Association for Information Science and Technology 65, 8: 1695-1706.

[10] Pierre Wellner. 1993. Interacting with paper on the digitaldesk. Communications of the ACM 36, 7: 87-96.

[11] Merkourios Margaritopoulos and Elissavet Georgiadou. 2019. The application of augmented reality in print media. Journal of Print and Media Technology Research 8: 43-55.

[12] Peter Ljungstrand and Lars Erik Holmquist. 1999. WebStickers: using physical objects as WWW bookmarks. CHI'99 Extended Abstracts on Human Factors in Computing Systems, 332-333.

[13] Scott R Klemmer, Jamey Graham, Gregory J Wolff, and James A Landay. 2003. Books with voices: paper transcripts as a physical interface to oral histories. Proceedings of the SIGCHI conference on human factors in computing systems, 89-96.

[14] Ron Yeh, et al. 2006. ButterflyNet. Proceedings of the SIGCHI conference on Human Factors in computing systems - CHI '06, ACM Press, 571.

[15] Mark Billinghurst, Hirokazu Kato, and Ivan Poupyrev. 2001. The magicbook-moving seamlessly between reality and virtuality. IEEE Computer Graphics and applications 21, 3: 6-8.

[16] David West, Aaron Quigley, and Judy Kay. 2007. MEMENTO: a digital-physical scrapbook for memory sharing. Personal and Ubiquitous Computing 11, 4: 313-328

[17] Christopher Baume, Mark Plumbley, David Frohlich, and Janko Calic. 2018. PaperClip: A digital pen interface for semantic speech editing in radio production. Journal of the Audio Engineering Society 66, 4: 241-252.

[18] David M Frohlich, Tony Clancy, John Robinson, and Enrico Costanza. 2004. The audiophoto desk. Proc. 2AD Demos: 139.

[19] M. Back, et al. 2001. An electronically augmented paper-based book. Conference on Human Factors in Computing Systems - Proc.: $23-29$.

[20] Paul Luff, et al. 2007. Augmented paper: developing relationships between digital content and paper. In The disappearing computer. Springer, 275-297.

[21] David M. Frohlich, et al. 2017. Designing interactive newsprint. International Journal of Human Computer Studies 104, July 2015: 36-49.

[22] Gavin Bailey, Deepak Sahoo, and Matt Jones. 2017. Paper for E-paper: Towards paper like tangible experience using E-paper. Proceedings of the 2017 ACM International Conference on Interactive Surfaces and Spaces, 446-449.

[23] Iyubanit Rodriguez, Simón Rodriguez, Andres Lucero, and Valeria Herskovic. 2019. TorBook: a tangible book for older adults. Proceedings of the IX Latin American Conference on Human Computer Interaction, 1-6.

[24] University of Surrey. Light Tags. Retrieved January 5, 2021 from https://www.surrey.ac.uk/digital-world-research-centre/fundedprojects/light-tags.

[25] Radu A. Sporea, et al. 2018. Next generation paper: an augmented book platform. Organic and Hybrid Sensors and Bioelectronics XI, SPIE, 37.

[26] David M. Frohlich, Emily Corrigan-Kavanagh, Megan Beynon, et al. 2019. The Cornwall a-book: An Augmented Travel Guide Using Next Generation Paper. The Journal of Electronic Publishing 22, 1: 1-9.

[27] Emily Corrigan-Kavanagh, Caroline Scarles, and George Revill. 2019. Augmenting Travel Guides for Enriching Travel Experiences. e-Review of Tourism Research 17: 344-348.

[28] Gavin Bailey, Deepak Ranjan Sahoo, and Matt Jones. 2020. Digital Bookmark: Seamless Switching Between Printed and Electronic Books. Proceedings of the 2020 ACM Designing Interactive Systems Conference, 885-894. 